\title{
Análise da distribuição espacial de larvas de Aedes aegypti na Ilha do Governador, Rio de Janeiro, Brasil
}

\author{
Spatial analysis of Aedes aegypti larval \\ distribution in the Ilha do Governador \\ neighborhood of Rio de Janeiro, Brazil
}

Reinaldo Souza-Santos 1

Marilia Sá Carvalho 2

\footnotetext{
1 Departamento de Endemias Samuel Pessoa, Escola

Nacional de Saúde Pública Fundação Oswaldo Cruz.

Rua Leopoldo Bulhões 1480 térreo, Rio de Janeiro, RJ 21045-900, Brasil. rssantos@ensp.fiocruz.br. 2 Departamento de Epidemiologia e Métodos Quantitativos em Saúde, Escola Nacional de Saúde Pública, Fundação Oswaldo Cruz. Rua Leopoldo Bulhões 1480 8 o andar, Rio de Janeiro, $R J$ 21045-900, Brasil.
}

\begin{abstract}
This study aims to help expand knowledge on Aedes aegypti in Rio de Janeiro, based on spatial analysis of the mosquito's breeding sites in the neighborhood of Ilha do Governador, Rio de Janeiro, from June 1992 to July 1994. Use of spatial analysis techniques is proposed for vector surveillance and control. Information obtained from the National Health Foundation shows which city blocks were positive for A. aegypti larvae and the number of dwellings per block that were used for the analysis. A smoothing method, a Gaussian Kernel, was used in the spatial distribution analysis. Breaks in National Health Foundation intervention activities were observed between cycles 4 and 5 and 5 and 6, respectively, followed by increases in vector density and vector-positive areas. Permanently positive areas, like slums and a military compound, display the persistence of favorable environmental conditions for oviposition and larval growth, indicating flaws in the vector control program. Although Kernel is an exploratory analytical method involving subjective interpretation, it provides easy and fast visualization of sites subject to different degrees of risk, unaffected by existing political and administrative territorial apportionment.
\end{abstract}

Key words Aedes aegypti; Dengue; Vector Control; Spatial analysis

Resumo Este trabalho visa contribuir para o conhecimento sobre Aedes aegypti no Rio de Janeiro com base na análise espacial de seus criadouros na Ilha do Governador, Rio de Janeiro, entre junho de 1992 e julho de 1994. Propõe a utilização de técnicas de análise de dados espaciais na vigilância e controle desse vetor. Utilizaram-se informações obtidas na Fundação Nacional de Saúde (FNS) que relatam em quais quarteirões foram encontradas larvas de A. aegypti $e o$ número de residências por quarteirão. Na análise espacial, utilizou-se o método Kernel gaussiano. Entre o ciclo 4 e 5 e entre o 5 e 6, observam-se intervalos de atividades da FNS, seguidos de aumento da densidade de quarteirões positivos e da abrangência de suas áreas. Locais permanentemente positivos, como a região de favela e a vila militar 1, mostram a manutenção de ambientes propícios para ovipostura e crescimento de larvas, indicando falhas no controle do vetor. Embora Kernel seja um método de análise exploratória de interpretação subjetiva, possibilita fácil e rápida visualização de localidades expostas a diferentes graus de risco, sem serem afetadas pelas divisões político-administrativas existentes.

Palavras-chave Aedes aegypti; Dengue; Controle de Vetores; Análise Espacial 


\section{Introdução}

O número de municípios brasileiros nos quais tem sido assinalada a presença de Aedes aegypti, vetor da dengue e da febre amarela, tem aumentado rapidamente nos últimos anos -1.802 em 1995, 2.110 em 1996 e 2.714 em 1997, evidenciando dificuldades dos programas e ações de controle (Nobre et al., 1994; Silveira, 1998). Atualmente, esse vetor pode ser encontrado em todos os estados do Brasil. Como esperado, a casuística da dengue acompanha a tendência à dispersão de A. aegypti no território nacional: 128.619 casos em 1995, 183.418 em 1996 e 254.942 em 1997 (Merchán-Hamann, 1997; Silveira, 1998). Apesar do grande número de estudos realizados em diferentes países (Focks et al., 1981; Kemp \& Jupp, 1991; Ko et al., 1992; Edgerly et al., 1993; Focks et al., 1993; Schultz, 1993; Reiter et al., 1995; Tun-Lin et al., 1995; Chadee et al., 1998; Russell, 1998; dentre outros) que visam ao melhor conhecimento da biologia, ecologia e controle de A. aegypti, no Brasil a espécie tem sido relativamente pouco investigada, considerando-se a heterogeneidade de ambientes nos quais ocorre em todo o País.

A utilização de técnicas que visam à análise da distribuição espacial de insetos vetores objetivando seu monitoramento e controle vem se ampliando nos últimos anos em diversos países (Ritchie, 1993; Beck et al., 1994; Kitron et al., 1994; Smith et al., 1995; Hay, 1997; Dale et al., 1998; Spradling et al., 1998). Vale ressaltar que a quase totalidade desses estudos versa sobre o gênero Anopheles, com predominância de investigações realizadas no continente africano. Desconhece-se a existência de estudos que façam uso de metodologias de análise espacial direcionadas ao monitoramento e controle de A. aegypti.

No que pese o crescente interesse no Brasil por estudos epidemiológicos que utilizam técnicas de análise espacial (Medronho, 1995; Bretas \& Bessa, 1996; Nobre et al., 1996; Soares Filho \& Sawyer, 1996), a ênfase da totalidade dos trabalhos disponíveis recai sobre a distribuição de casos de doenças em si, pouco ou nada sendo dito a respeito das populações de vetores.

O presente trabalho visa contribuir para o maior conhecimento sobre A. aegypti no Rio de Janeiro, com base em um estudo conduzido na Ilha do Governador, Rio de Janeiro, onde se propõe a utilização de técnicas de análise de dados espaciais na vigilância e controle desse vetor.

\section{Material e métodos}

\section{Área de estudo}

A área de estudo compreende o bairro do Galeão, localizado na Ilha do Governador, Rio de Janeiro. Esse bairro possui $20,28 \mathrm{~km}^{2}$, que corresponde a quase metade da área total da Ilha, que encontra-se localizada no interior da Baía de Guanabara. Nele se encontram, além do Aeroporto Internacional do Rio de Janeiro, setores urbanizados, favelas e áreas de vegetação secundária. Os prédios existentes no perímetro do aeroporto não foram considerados, pois o serviço de vigilância e controle de vetores em portos e aeroportos é desenvolvido por um setor especializado da Fundação Nacional de Saúde (FNS), que não gerou dados possíveis de serem utilizados na presente análise. Em virtude da disposição dos quarteirões no perímetro do bairro, foram atribuídas letras às zonas não contíguas e conectadas por via rodoviária ( $\mathrm{Fi}$ gura 1). Ao todo, foram consideradas quatro zonas: A - constituída por prédios da INFRAERO (Empresa Brasileira de Infra-Estrutura Aeroportuária), situada entre a pista do aeroporto e a praia de Tubiacanga; $\mathrm{B}$ - região residencial que se encontra na Ponta de Tubiacanga, próxima à praia e à pista de pouso e decolagem do aeroporto, com pequenos pontos de vegetação; C - a maior, constituída por duas vilas militares, aqui denominadas 1 e 2, com prédios residenciais e casas, estabelecimentos comerciais, um posto de gasolina, galpões da INFRAERO e uma central elétrica, além de uma região favelada denominada Vila Joaniza (Morro do Barbante), com extensos canteiros contíguos à estrada de acesso ao aeroporto; D - perímetro comercial constituído pelo terminal de cargas do aeroporto, depósito de cargas dos Correios e prédios da INFRAERO.

O número de habitantes, de acordo com o censo demográfico de 1991, era de 17.562 pessoas, com densidade bruta de 8,7 habitantes/ ha. Do total de 4.354 domicílios, $92,8 \%$ possuem canalização interna de água, 91,4\% estão ligados à rede geral de abastecimento de água, $96 \%$ possuem canalização interna de esgotos, $75,5 \%$ estão conectados à rede geral de esgoto e $76,7 \%$ possuem lixo coletado diretamente (IPLANRIO, 1995). As médias anuais de temperatura são de $26,7 \circ \mathrm{o}$ (máxima) e 20,4ㄷ (mínima). A pluviosidade média anual é de 1.107 $\mathrm{mm}$, com freqüência média de 124 dias de chuva por ano (IPLANRIO, 1995). 
Figura 1

Bairro do Galeão, Ilha do Governador, Rio de Janeiro, Brasil.

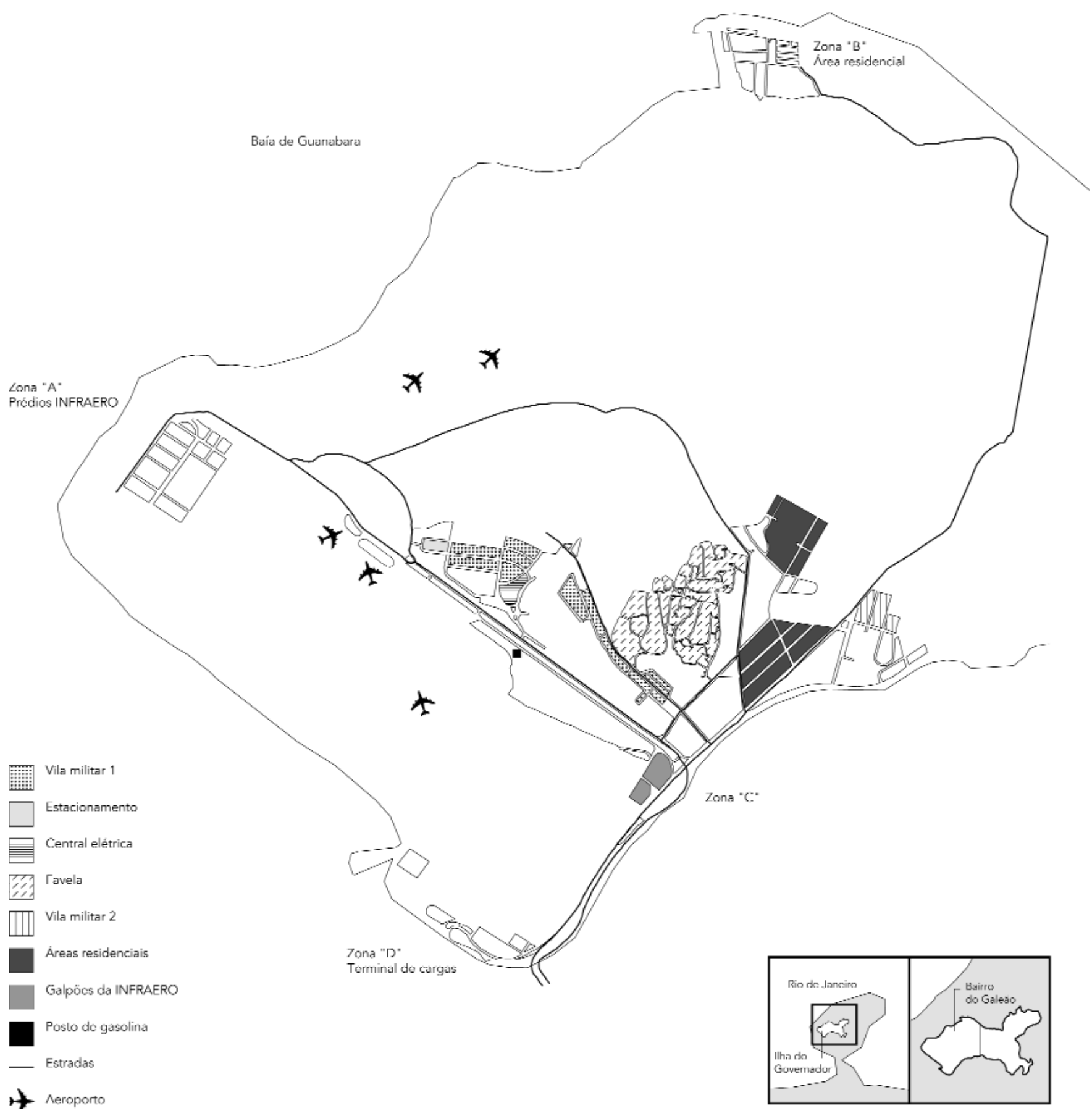




\section{Os dados}

O período coberto pelo estudo foi de junho de 1992 a julho de 1994. Para a análise, foram utilizadas informações obtidas em visitas domiciliares efetuadas pelos funcionários da FNS, que relatam quais quarteirões estão positivos para encontros de larvas de A. aegypti e o número de residências e/ou estabelecimentos por quarteirão.

Obteve-se também, junto à FNS, o croqui do bairro do Galeão. Este foi digitalizado em metros, com seus 124 quarteirões devidamente numerados. O banco de dados, com o número dos quarteirões positivos ou não, e o total de residências e/ou estabelecimentos, foi georreferenciado. Assim, todas as informações contidas no banco de dados puderam ser desenhadas no mapa, utilizando as coordenadas dos quarteirões.

As atividades da FNS no bairro do Galeão foram efetuadas em seis ciclos, cada qual com duração média de três meses, e com um intervalo entre eles variando de uma semana a três meses. Dessa forma, apesar de os dados não serem contínuos, a base de dados da FNS dispõe de informações relativas a seis ciclos de levantamentos, referentes aos seguintes períodos: primeiro ciclo - de 15/6/92 a 16/9/92; segundo ciclo - de 5/10/92 a 17/2/93; terceiro ciclo $-24 / 2 / 93$ a 11/6/93; quarto ciclo $-21 / 6 / 93$ a 20/8/93; quinto ciclo - 29/11/93 a $7 / 2 / 94$; sexto ciclo $-6 / 4 / 94$ a 28/7/94.

\section{A análise}

Utilizando-se o programa SPSS, versão 8.0, procedeu-se à análise descritiva dos dados referentes ao número de quarteirões por ciclo, positivos ou não para larvas de A. aegypti. A análise dos dados espaciais foi realizada empregando-se o programa S-plus, versão 3.4 com módulo espacial.

O método escolhido para a investigação do padrão de distribuição dos eventos localizados pontualmente (considerando as coordenadas do quarteirão) foi a interpolação e alisamento através de um Kernel gaussiano (Bailey \& Gatrell, 1995). Este permite estimar a probabilidade de ocorrência de um evento em cada cela de uma grade regular, sendo cada célula desta grade a média ponderada de todos os valores da vizinhança, cujos pesos são atribuídos utilizando uma função - nesse caso a gaussiana de distribuição de probabilidades. O grau de alisamento é controlado mediante a escolha de um parâmetro conhecido como largura de banda, que indica a área que será considerada no cálculo, e deve refletir a escala geográfica da hipótese de interesse e o conhecimento prévio sobre o problema estudado (Santos, 1999). Em nossa análise, utilizou-se largura de banda de 300 metros com base na dispersão das fêmeas de A. aegypti. Apesar de estas normalmente não voarem mais que cem metros, caso não existam criadouros apropriados no local, elas podem aumentar seu raio de dispersão á procura de um lugar para pôr seus ovos (Trpis et al., 1995).

Utilizaram-se duas formas de alisamento por Kernel: uma visando estimar a densidade de quarteirões positivos (Equação 1), e a outra, a densidade de habitações por quarteirão (Equação 2). A primeira dessas medidas permite visualizar a intensidade de infestação; a segunda estima a distribuição de residências. A razão das duas consiste em uma medida de risco para a transmissão de dengue.

Equação 1

$\hat{\lambda}(s)=\sum_{i=1}^{n} \frac{1}{\tau^{2}} k\left(\frac{\left(s-s_{i}\right)}{\tau}\right)$

Equação 2

$\hat{\lambda}(s)=\sum_{i=1}^{n} \frac{1}{\tau^{2}} k\left(\frac{\left(s-s_{i}\right)}{\tau}\right) y_{i}$

Onde,

$\lambda(\mathrm{s})$ - valor estimado por área;

$\tau$ - largura da banda (fator de alisamento);

$k$ ( ) - função de ponderação Kernel, no caso uma distribuição gaussiana;

$s$ - centro de cada espaço da grade regular;

$s_{\mathrm{i}}$ - local do ponto onde ocorreu o evento;

$y_{\mathrm{i}}$ - valor do evento no ponto (no de residências por quarteirão).

Como os dados da FNS mostraram uma pequena variação do total de residências e/ou estabelecimentos por quarteirão, essa contagem no ciclo 2 foi a escolhida para a estimativa de densidade de residências, por apresentar o maior número de construções. Um quarteirão que se localizava próximo à zona $\mathrm{D}$ foi excluído, uma vez que nele se encontrava apenas um galpão pertencente à INFRAERO negativo em todos os ciclos (Figura 1). A estimativa foi feita considerando a área inteira. Como, entretanto, esta é formada por quatro polígonos relativamente distantes entre si, a apresentação foi feita excluindo os espaços entre as zonas.

Utilizou-se a denominação de densidade baixa, média e alta para a análise dos quarteirões e a do indicador de risco, uma vez que a primeira traduz a intensidade de quarteirões 
positivos, e a segunda, a intensidade de risco de transmissão de dengue. Adotaram-se os menores valores, ou áreas mais claras, para aqueles quarteirões com menor densidade ou risco de transmissão de dengue, uma vez que existem relatos de epidemia da doença em locais com índice de infestação predial de 1\% (OPS, 1995).

\section{Resultados}

Com base nas datas de início e fim de cada ciclo, calculou-se o número de semanas trabalhadas para cada um deles (Tabela 1). A média do número de dias trabalhados em cada semana foi de 6. Observou-se também que, entre os ciclos 4 e 5 e entre os ciclos 5 e 6 , houve um intervalo de três e dois meses, respectivamente.

Ao observar-se o total de quarteirões com criadouros positivos por ciclo (Tabela 1), notase menor percentual para o ciclo $4(3,5)$ e os maiores para os ciclos $5(27,8)$ e $6(40,9)$. Apesar do elevado percentual no ciclo $1(32,8)$, deve-se levar em consideração que, neste ciclo, o número de quarteirões inspecionados foi de 64 , estando bem abaixo dos inspecionados nos outros ciclos.

\section{Densidade de quarteirões positivos para larvas de A. aegypti, método Kernel (Figura 2)}

A análise para o ciclo 1 mostra que não houve ocorrência de quarteirões positivos nas zonas A e B. Porém, na zona C, observa-se densidade média de quarteirões positivos em toda a área da vila militar 1 , na central elétrica e em alguns grupamentos de casas em pontos distintos do bairro. Uma maior densidade de quarteirões positivos, sem alcançar a máxima, pode ser observada na área mais ao norte da região favela- da. Densidade baixa é observada na vila militar 2, o mesmo ocorrendo na zona D, no local do terminal de cargas do aeroporto.

No ciclo 2, observa-se o surgimento de quarteirões positivos nas zonas A e B, apresentando baixa densidade no extremo norte da zona A e em quase toda a zona B. Na realidade, no ciclo 1, os quarteirões dessas zonas não foram inspecionados pela equipe da FNS. O número de quarteirões inspecionados no primeiro ciclo corresponde a $64 \%$ daqueles que o foram no segundo ciclo. Assim, não se pode fazer nenhuma inferência acerca dessas duas zonas entre esses dois ciclos. Na zona C, verifica-se uma redução da área com quarteirões positivos, com ocorrência de baixa densidade no terreno utilizado como estacionamento para carros de aluguel e em parte da vila militar 1 . Notam-se, ainda, dois picos de maior densidade de quarteirões positivos, contudo não alcançando a máxima, nas regiões da favela e da vila militar 1. A zona D continua como no ciclo anterior, apresentando baixa densidade.

$\mathrm{O}$ ciclo 3 caracteriza-se por pequenos pontos de baixa densidade de quarteirões nas zonas A e B, como no ciclo 2. A zona C apresenta um aumento tanto na região com quarteirões positivos, quanto na densidade na área da favela e da vila militar 1 . Observa-se, também, que os pontos com baixa densidade são correspondentes àqueles do ciclo 2 , acrescidos pelo posto de gasolina, galpões da INFRAERO, vila militar 2 e região residencial. Semelhante ao ciclo anterior, a zona D permanece com baixa densidade.

No ciclo 4, identificamos apenas três pontos com baixa densidade na zona $\mathrm{C}$, sendo um correspondente a um ponto da vila militar 1 , outro na periferia da favela e o terceiro na periferia da vila militar 2. A zona D apresenta-se da mesma forma que nos ciclos anteriores, com baixa densidade de quarteirões positivos.

Número de quarteirões com criadouros positivos para A. aegypti por ciclo de trabalho da Fundação Nacional de Saúde, no bairro do Galeão, Rio de Janeiro, 1992-1994.

\begin{tabular}{lcccrrr}
\hline & Ciclo 1 & Ciclo 2 & Ciclo 3 & Ciclo 4 & Ciclo 5 & Ciclo 6 \\
\hline Número de semanas & 14 & 20 & 16 & 9 & 8 & 17 \\
Quarteirões inspecionados (n) & 64 & 119 & 120 & 114 & 115 & 115 \\
Quarteirões positivos (n) & 21 & 14 & 29 & 4 & 32 & 47 \\
\% & 32,8 & 11,8 & 24,2 & 3,5 & 27,8 & 40,9 \\
\hline
\end{tabular}

Obs: Quarteirões existentes: $n=124$ 
Figura 2

Densidade de quarteirões positivos para larvas de A. aegypti, método Kernel. Bairro do Galeão, Ilha do Governador, Rio de Janeiro, Brasil, de junho de 1992 a julho de 1994.
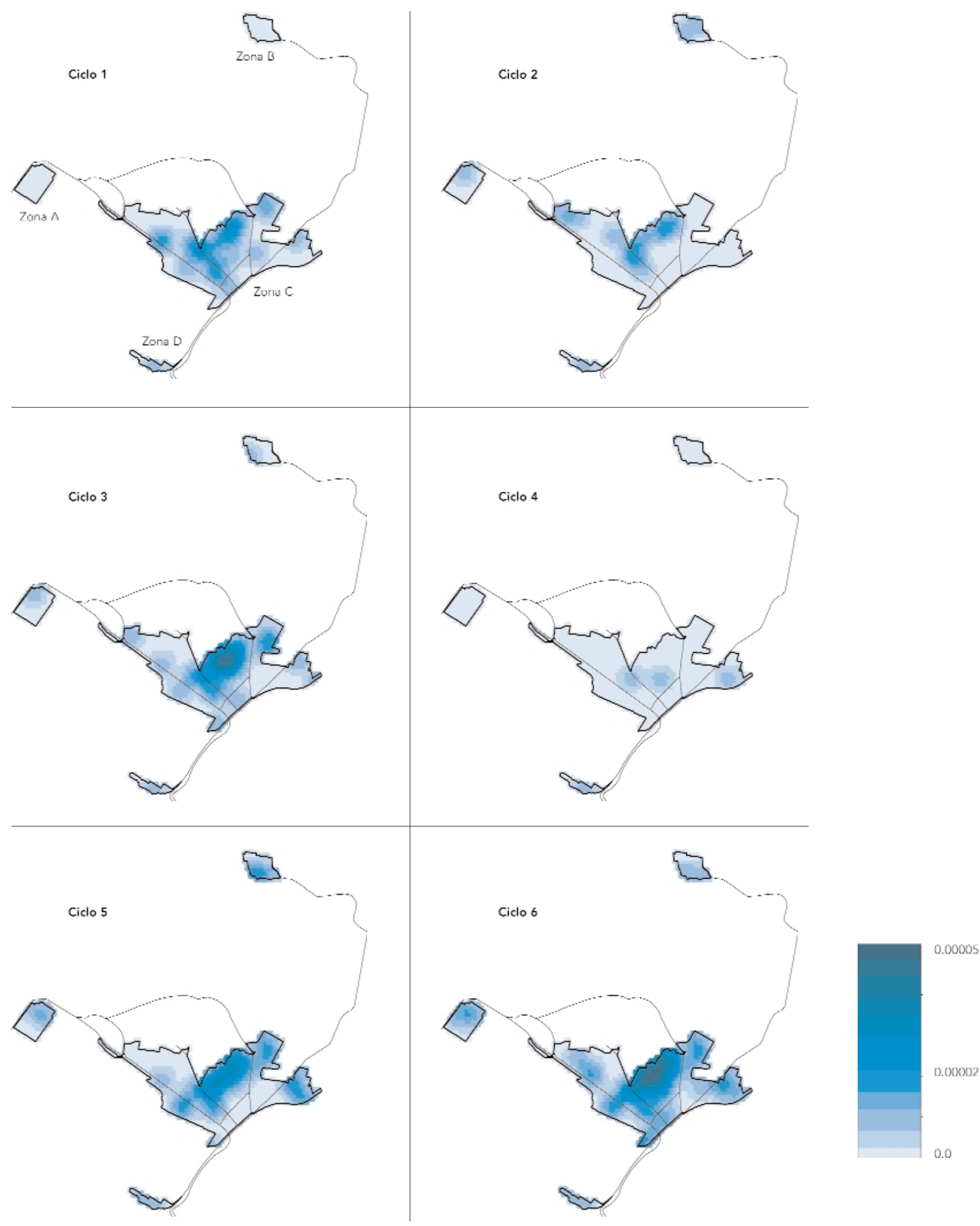


\section{Figura 3}

Densidade de risco para transmissão da dengue, método Kernel. Bairro do Galeão, Ilha do Governador,

Rio de Janeiro, Brasil, de junho de 1992 a julho de 1994.
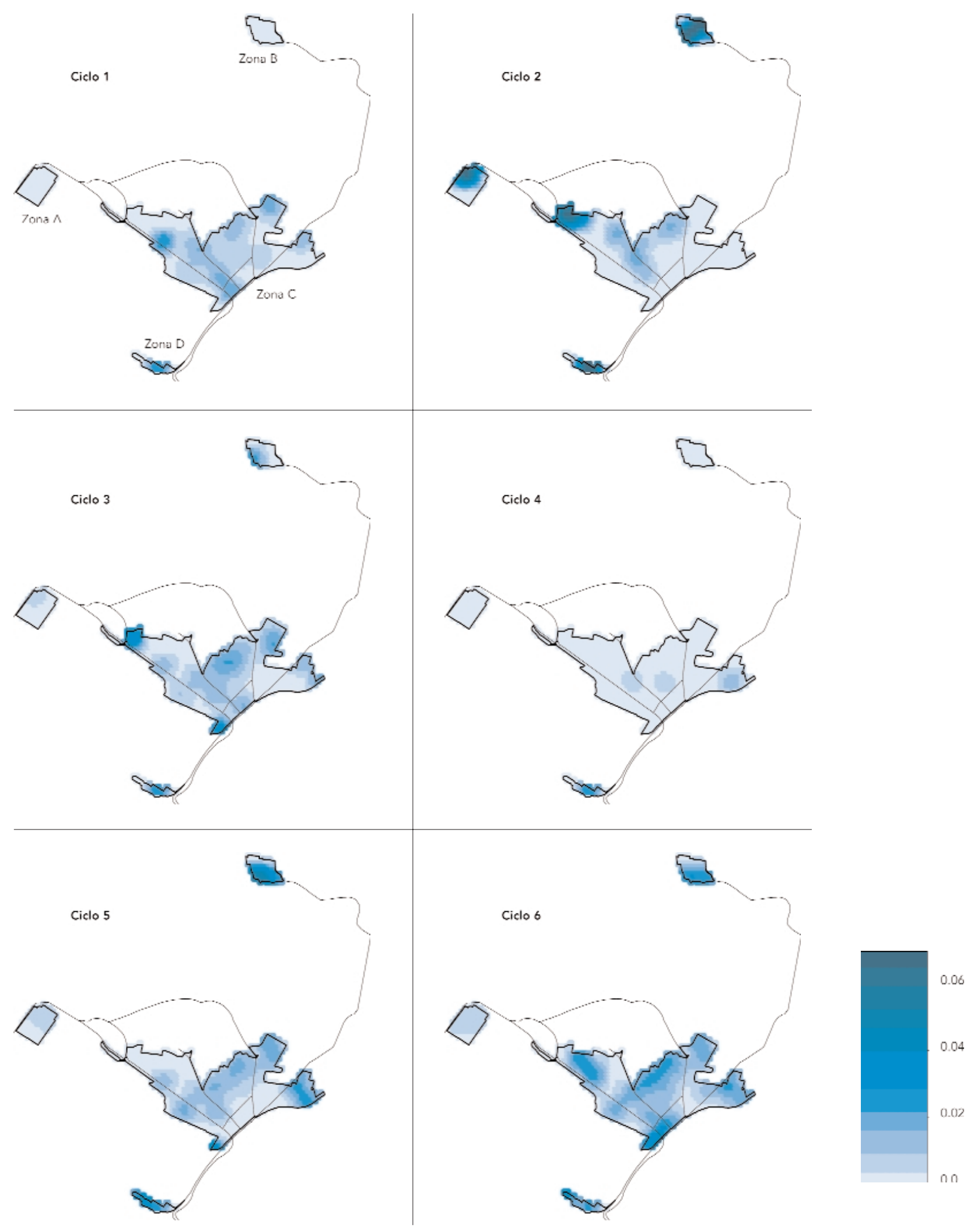
O resultado do Kernel para o ciclo 5 mostra a zona A com densidade baixa. A zona B apresenta um pequeno ponto com densidade média e quase todo o restante da zona com densidade baixa. Na zona C, praticamente toda a região da favela encontra-se com densidade alta, não alcançando a máxima, e formando um contínuo entre a segunda e parte da vila militar 1. Além disso, diversos pontos, tais como as vilas militares, o posto de gasolina e região residencial apresentam densidades médias. Com baixa densidade, foram identificados os galpões da INFRAERO e a central elétrica. A zona D mostra baixa densidade em praticamente toda a sua área.

No ciclo 6, foi detectado um ligeiro aumento na densidade e na região positiva da zona A, ou seja, apresentando um ponto com densidade média e todo o restante com baixa. Na zona $\mathrm{B}$, houve redução na densidade, que se mostrou baixa ao sul e reduzindo ao norte. Verificase que, em relação ao ciclo anterior, a zona $C$ apresenta maior densidade em diversos locais, além de um aumento na área com quarteirões positivos. A favela pode ser identificada como a região de densidade máxima, formando um contínuo com parte da vila militar 1 . O posto de gasolina, as vilas militares, as regiões residenciais, os galpões da INFRAERO e a central elétrica apresentam densidade média. Novamente a zona D mostra-se com baixa densidade em praticamente toda sua área.

\section{Densidade de risco para transmissão da dengue, método Kernel (Figura 3)}

A análise do ciclo 1 mostra as zonas A e B sem nenhum risco. A zona $\mathrm{C}$ apresenta densidade média no local da central elétrica e na entrada da vila militar 1 , no ponto mais ao norte da favela, na vila militar 2 e em quarteirões residenciais. Além disso, nota-se uma redução da área exposta ao risco de ocorrência de dengue. Parte da zona D, onde se localiza o terminal de cargas do aeroporto, apresenta densidade média.

No ciclo 2, verifica-se baixa densidade na zona A e densidade média na B. Na análise da zona $\mathrm{C}$, identifica-se densidade máxima no local do estacionamento para automóveis de aluguel. A favela e a vila militar 1 apresentam densidades baixas. Como no ciclo anterior, uma porção da zona D caracteriza-se por densidade média.

Ao analisar-se o ciclo 3, nota-se que a zona A continua com baixa densidade. A zona B apresenta densidade média, com abrangência menor que no ciclo anterior. A zona C evidencia um aumento na área de risco, que se carac- teriza por: dois pontos com densidade alta (galpões da INFRAERO e o terreno para carros de aluguel), dois pontos com densidade média (favela e região residencial) e as vilas militares, a central elétrica, o posto de gasolina e uma região residencial com densidade baixa. Parte da zona D continua com densidade média.

A análise da densidade de risco para o ciclo 4 mostra apenas três pontos com baixa densidade na zona $\mathrm{C}$, localizados na vila militar 1 , na periferia da favela e próximo à vila militar 2. Da mesma forma que os ciclos anteriores, parte da zona $\mathrm{D}$ apresenta densidade média.

No ciclo 5, pode-se identificar a zona A com baixa densidade apenas ao norte, e a zona B com densidade máxima ao sul, reduzindo nos sentidos norte e leste. A zona C apresenta-se com densidade média em três extremos: quarteirões residenciais, a vila militar 2 e os galpões da INFRAERO. A região da favela, a vila militar 1 , a central elétrica e o posto de gasolina mostram-se com densidade baixa. Observam-se densidades médias em praticamente toda a zona D.

Com base nos resultados para o ciclo 6, verifica-se um grande aumento da região exposta ao risco em todas as zonas. Essa mesma ocorrência é notada na zona $A$, que se apresenta quase totalmente com baixa densidade. Na zona $B$, observa-se que quase toda a área encontra-se sob risco de densidade média. Ao analisar-se a zona C, constata-se que a região da favela, a central elétrica e adjacências mostram densidade média. Os quarteirões residenciais, as vilas militares e o posto de gasolina apresentam densidade baixa, enquanto os galpões da INFRAERO apresentam densidade alta. A zona D mostra-se com densidade média em toda sua área.

\section{Densidade de quarteirões positivos versus a densidade de risco para transmissão de dengue}

A comparação dos resultados da densidade de quarteirões positivos e da densidade de risco para transmissão da dengue (Figura 2 e 3, respectivamente) mostra, como era de se esperar, que as regiões que apresentaram densidade positiva, nas duas figuras, são as mesmas. Observam-se diferenças nas densidades em determinadas zonas. A comparação das análises para cada ciclo nos mostra o seguinte:

- Ciclo 1 - A vila militar 1 e a favela, que apresentavam densidade média para quarteirões positivos, possuem apenas risco médio em alguns pontos, mostrando a área restante com risco baixo. A central elétrica e as zonas resi- 
denciais apresentam risco médio; já a vila militar 2, risco baixo, mostrando resultados semelhantes aos encontrados para as densidades de quarteirões positivos.

- Ciclo 2 - A zona A, que apresentava apenas baixa densidade de criadouros positivos, mostra baixo risco para transmissão. Já a zona B, que tinha uma densidade baixa, apresenta risco médio. Observa-se que, na zona $\mathrm{C}$, o terreno utilizado como estacionamento para carros de aluguel mostra densidade baixa para quarteirões positivos, verificando-se risco máximo para transmissão de dengue. Na zona da favela e da vila militar 1, que apresentavam pontos com alta densidade, identifica-se baixo risco para transmissão de dengue. Na zona $\mathrm{D}$, verifica-se risco médio, em contraste com a baixa densidade de quarteirões positivos.

- Ciclo 3 - Na zona A, verifica-se baixa densidade para quarteirões positivos e baixo risco. A comparação dos resultados para a zona B mostra-nos baixa densidade para quarteirões positivos e risco médio. A zona $\mathrm{C}$ apresenta baixa densidade e alto risco no terreno utilizado para estacionamento de carros de aluguel e nos galpões da INFRAERO, alta densidade e risco médio na favela e em uma zona residencial, baixa densidade e risco baixo para o posto de gasolina, vila militar 2 e central elétrica e, finalmente, densidade média e risco baixo para a vila militar 1. A zona D apresenta-se como no ciclo anterior, com baixa densidade e risco médio.

- Ciclo 4 - Observam-se, na zona C, os três pontos - vila militar 1 , periferia da favela e periferia da vila militar 2 - com baixa densidade e baixo risco. A zona D apresenta-se como no ciclo anterior.

- Ciclo 5 - Na zona A, verifica-se baixa densidade e baixo risco apenas ao norte. A zona B apresenta um ponto com densidade média e quase todo o restante com baixa, enquanto a análise do indicador de risco mostra risco máximo ao sul, reduzindo nos sentidos norte e leste. Na zona C, identifica-se densidade alta em toda área da favela e parte da vila militar 1 , pois o restante desta apresenta densidade média. O indicador de risco mostra essas áreas com densidade baixa. A central elétrica apresenta baixa densidade e baixo risco, o posto de gasolina, densidade média e baixo risco, e os galpões da INFRAERO, densidade baixa e risco médio. A vila militar 2 e a área residencial apresentam densidade e risco médios. A zona D apresenta-se da mesma forma que no ciclo anterior, com baixa densidade e risco médio.

- Ciclo 6 - Observa-se a zona A com densidade e risco baixos. A zona B com baixa densidade e risco médio ao sul, reduzindo ao norte. $\mathrm{Na}$ zona $\mathrm{C}$, verifica-se densidade máxima e risco médio na região da favela. Nas vilas militares, posto de gasolina e áreas residenciais, constata-se densidade média e risco baixo. A central elétrica mostra densidade e risco médios, ao passo que os galpões da INFRAERO apresentam densidade média e risco alto. A zona D encontra-se como no ciclo anterior.

\section{Discussão}

Aedes aegypti é um mosquito com hábito predominantemente urbano (Consoli \& Lourenço-de-Oliveira, 1994). Suas larvas podem ser facilmente encontradas em diversos tipos de criadouros naturais e/ou artificiais no domicílio e/ou peridomicílio (Focks et al., 1981; Lopes et al., 1993; Chiaravalloti Neto, 1997; SouzaSantos, 1999). Neste trabalho, confirma-se a ocorrência de A. aegypti não apenas em zonas residenciais da Ilha do Governador (principalmente vilas militares e favela), mas também naquelas com fins comerciais, tais como galpões para armazenamento de cargas, postos de gasolina e estacionamento de automóveis. Apesar de as ocorrências de quarteirões positivos serem, principalmente, em área residencial, esse resultado não aponta uma tendência, pois, excetuando o aeroporto, o bairro do Galeão possui mais zonas residenciais do que comerciais.

Os dados da FNS mostram que o número de semanas trabalhadas não está diretamente relacionado ao número de quarteirões inspecionados. No ciclo 1, que foi trabalhado em 14 semanas, verificam-se 64 quarteirões inspecionados; no ciclo 4, com duração de nove semanas, há 114 quarteirões inspecionados, e, no ciclo 6, com 17 semanas, tem-se 115 quarteirões inspecionados. Como não se sabe quantos funcionários trabalharam em cada ciclo, torna-se difícil avaliar se houve uma variação no esforço/tempo gasto pela FNS em cada ciclo.

A evolução da densidade de quarteirões positivos para larvas de A. aegypti durante os seis ciclos (Figura 2) evidencia locais permanentemente positivos, como a região de favela e a vila militar 1. Segundo Sabroza et al. (1992), a impossibilidade de acesso de agentes do serviço público a determinadas áreas da cidade impede a cobertura adequada da região infestada visando à identificação e destruição dos focos das larvas. Além disso, a distribuição intermitente de água no sistema de abastecimento público em certas áreas, ou a total inexistência deste, leva a população a manter reservatórios improvisados sobre as lages das casas ou nos quintais, o que proporciona condições ideais à 
reprodução do vetor (Sabroza et al., 1992; Barcellos et al., 1998; Souza-Santos, 1999). A região da favela não somente constitui exemplo típico de zona de difícil acesso aos agentes de saúde, como também caracteriza-se pela irregularidade de suprimento de água potável. Quanto às vilas militares, acredita-se que sejam mantidas livres de criadouros provenientes de lixo doméstico, que possuam fornecimento regular de água e que sejam de acesso relativamente fácil para os agentes de saúde. Apesar disso, nossos achados indicam a existência de falhas no programa de controle deste vetor.

As diferenças observadas entre os seis ciclos, não apenas no que se refere à densidade de quarteirões positivos, mas também em relação à área de abrangência, resultam tanto da ação da FNS, quanto, principalmente, das mudanças climáticas e da "produção" de criadouros pela população (Souza-Santos, 1999). Não obstante o reduzido número de quarteirões inspecionados no ciclo 1, nota-se, em relação ao ciclo 2, uma diminuição da área com quarteirões positivos na zona C. Ao comparar o segundo ciclo com o seguinte, ciclo 3, percebe-se um aumento tanto da área positiva, como da densidade em algumas localidades, como a região favelada. No ciclo 4, observamos uma acentuada redução das localidades positivas e de suas áreas. Contudo, vale ressaltar, essas localidades correspondem às vilas militares e a um ponto na periferia da favela. Entre os ciclos 4 e 5 e entre o 5 e 6 , houve um intervalo de atividades da FNS de três e dois meses, respectivamente. Subseqüente ao primeiro intervalo, observou-se, no ciclo 5, densidade alta em toda a região da favela e parte da vila militar 1 , além de um aumento de sua abrangência. Após o segundo intervalo, no ciclo 6 , notam-se praticamente todas as zonas com suas áreas positivas, além de um aumento na densidade, alcançando a máxima em algumas localidades. Esses achados mostram que a interrupção nas atividades de controle de A. aegypti, nos períodos descritos, fez com que a população deste vetor ultrapassasse os níveis anteriormente encontrados, o que é freqüente quando se verifica um relaxamento no controle dele (Mitchell, 1996).

A análise da Figura 2 mostra o surgimento, em determinados ciclos, de quarteirões positivos não contíguos àqueles previamente positivos. Após o repasto sangüíneo, a fêmea desloca-se em busca de criadouros para ovipostura. Ainda que seja raro um raio de dispersão de $A$. aegypti acima de cem metros, existe a possibilidade de transporte passivo de fêmeas, seja com auxílio do vento, ou por meio de veículos automotores. Além disso, deve-se ter em mente que os ovos podem ficar latentes por aproximadamente um ano, caso não haja água e/ou temperatura favorável para eclosão (OPS, 1995). Tanto a dispersão de fêmeas, quanto a latência dos ovos podem ser utilizados para justificar uma reinfestação ou surgimento de novos quarteirões positivos. Porém, o mais importante é que esses fatos indicam falhas nas atividades de controle de A. aegypti, resultante, em parte, das falhas de continuidade do programa.

\section{Indicador de risco para transmissão de dengue}

Partindo do pressuposto de que a Vigilância Entomológica atua com o conceito de risco, a distribuição e densidade dos vetores são parâmetros fundamentais para alcançar esse objetivo. A escolha do indicador que reflita um estado de transmissão deve contemplar a condição de reprodutividade, representatividade operacional e custo-benefício compatível (Gomes, 1999).

Existem vários índices utilizados para estimar a densidade e distribuição de A. aegypti e indicar o grau de risco para transmissão de dengue e febre amarela (OPS, 1995; Gomes, 1999). Apesar do emprego rotineiro desses, a estimativa do risco de dengue com base em seus resultados é questionável (Tun-Lin et al., 1995; Gomes, 1999).

Os resultados de nossas análises apontam para uma vantagem em relação aos indicadores normalmente usados: a densidade do risco de transmissão para dengue pode ser visualizada como um contínuo de diferentes tonalidades (Figura 3), sem as usuais divisões (no caso quarteirões), aproximando a interpretação do que ocorre na realidade, pois, para os mosquitos, os limites político-administrativos obviamente não existem.

A comparação entre a densidade de criadouros positivos e a densidade de risco para transmissão de dengue evidencia a importância da utilização de um parâmetro ponderador, que indique o número de indivíduos que habitam determinada localidade. Neste estudo, ao se ponderar a densidade de quarteirões positivos pelo número de residências existentes verifica-se que, no ciclo 6 , apesar de a zona da favela possuir densidade máxima para criadouros positivos, os quarteirões que se encontram expostos a maior risco são aqueles ocupados pelos galpões da INFRAERO. Por isso, as ações de controle desenvolvidas pelos agentes da FNS devem ser intensificadas nas áreas de maior risco, não permitindo, todavia, que aque- 
las com risco menor fiquem sem qualquer atividade de controle. Como foi dito anteriormente, as localidades devem ser identificadas com base em indicadores de risco e não apenas na densidade da população do vetor.

Os dados utilizados na análise deste estudo não são os ideais, já que não levam em consideração a quantidade de criadouros existentes nem a produtividade de cada um, por localidade. No entanto, a análise de dados espaciais aqui empregada mostra-se uma importante ferramenta na vigilância e controle não apenas de Aedes aegypti, mas de outros insetos vetores.

A interpretação dos resultados obtidos mediante a análise de Kernel é subjetiva, dependendo principalmente do conhecimento do pesquisador no que se refere ao evento estudado e à área em questão. Apresenta como maior vantagem a fácil e rápida visualização de localidades expostas a diferentes graus de risco,

\section{Agradecimentos}

Os autores agradecem à Coordenadoria Regional da Fundação Nacional de Saúde no Rio de Janeiro, pelo acesso às informações referentes ao vetor, e ao Prof. Dr. Carlos E. A. Coimbra Jr., pela leitura crítica dos originais e sugestões.

\section{Referências}

BAILEY, T. C. \& GATRELL, A. C., 1995. Interactive Spatial Data Analysis. Essex: Longman.

BARCELlOS, C.; COUTINHO, K.; PINA, M. F.; MAGALHÃES, M. M. A. F.; PAOLA, J. C. M. D. \& SANTOS, S. M., 1998. Inter-relacionamento de dados ambientais e de saúde: Análise de risco à saúde aplicada ao abastecimento de água no Rio de Janeiro utilizando Sistemas de Informações Geográficas. Cadernos de Saúde Pública, 14:597-605.

BECK, L. R.; RODRIGUEZ, M. H.; DISTER, S. W.; RODRIGUEZ, A. D.; REJMANKOVA, E.; ULLOA, A.; MEZA, R. A.; ROBERTS, D. R.; MICHAEL, A. S.; WASHINO, R. K.; HACKER, C. \& LEGTERS, L. J., 1994. Remote sensing as a landscape epidemiologic tool to identify villages at high risk for malaria transmission. American Journal of Tropical Medicine and Hygiene, 51:271-280.

BRETAS, G. \& BESSA, R., 1996. Um sistema geográfico de informações para o controle de malária na Amazônia. Informe Epidemiológico do SUS, 5:7381.

CHADEE, D. D.; WARD, R. A. \& NOVAK, R. J., 1998. Natural habits of Aedes aegypti in Caribbean - A review. Journal of the American Mosquito Control Association, 14:5-11. sem serem afetadas pelas divisões político-administrativas existentes, bem como a possibilidade de criação de indicadores de riscos pautados em outros parâmetros.

A existência de criadouros e/ou quarteirões positivos para larvas de A. aegypti na Ilha do Governador não é apenas propiciada por características locais, tais como fornecimento de água encanada e tipo de construção das habitações. A descontinuidade nas ações de controle apresenta-se como um importante fator na manutenção desse vetor. Vale ressaltar a necessidade de estudos mais consistentes em áreas urbanas, que possibilitem uma melhor compreensão dos fatores que possam influenciar na ocorrência e distribuição de A. aegypti e que orientem as ações de controle. Deve-se também levar em consideração o papel da população, tanto no controle, quanto na criação e/ou manutenção de possíveis criadouros.
CHIARAVALLOTI NETO, F., 1997. Conhecimentos da população sobre dengue, seus vetores e medidas de controle em São José do Rio Preto, São Paulo. Cadernos de Saúde Pública, 13:447-453.

CONSOLI, R. A. G. B. \& LOURENÇO-DE-OLIVEIRA, R., 1994. Principais Mosquitos de Importância Sanitária no Brasil. Rio de Janeiro: Editora Fiocruz.

DALE, P. E. R.; RITCHIE, S. A.; TERRITO, B. M.; MORRIS, C. D.; MUHAR, A. \& KAY, B. H., 1998. An overview of remote sensing and GIS for surveillance of mosquito vector habitats and risk assessment. Journal of Vector Ecology, 23:54-61.

EDGERLY, J. S.; WILLEY, M. S. \& LIVDAHL, T. P., 1993. The community ecology of Aedes egg hatching: Implications for a mosquito invasion. Ecological Entomology, 18:123-128.

FOCKS, D. A.; SACKETT, S. R.; BAILEY, D. L. \& DAME, D. A., 1981. Observations on container-breeding mosquitoes in New Orleans, Louisiana, with an estimate of the population density of Aedes aegypti (L.). American Journal of Tropical Medicine and Hygiene, 30:1329-1335.

FOCKS, D. A.; HAILE, D. G.; DANIELS, E. \& MOUNT, G. A., 1993. Dynamic life table model for Aedes aegypti (Diptera: Culicidae): Simulation results 
and validation. Journal of Medical Entomology, 30:1018-1028.

GOMES, A. C., 1999. Medidas dos níveis de infestação urbana para Aedes (Stegomyia) aegypti e Aedes (Stegomyia) albopictus em programa de vigilância entomológica. Informe Epidemiológico do SUS, 7:49-57.

HAY, S. I., 1997. Remote sensing and disease control: Past, present and future. Transactions of the Royal Society of Tropical Medicine and Hygiene, 91: 1005-1006.

KEMP, A. \& JUPP, P. G., 1991. Potential for dengue in South Africa: Mosquito ecology with particular reference to Aedes aegypti. Journal of the American Mosquito Control Association, 7:574-583.

KITRON, U.; PENER, H.; COSTIN, C.; ORSHAN, L.; GREENBERG, Z. \& SHALON, U., 1994. Geographic information system in malaria surveillance: Mosquito breeding and imported cases in Israel, 1992. American Journal of Tropical Medicine and Hygiene, 50:550-556.

KO, Y. C.; CHEN, M.-J. \& YEH, S. M., 1992. The predisposing and protective factors against dengue virus transmission by mosquito vector. American Journal of Epidemiology, 136:214-220.

LOPES, J.; SILVA, M. A. N.; BORSATO, A. M.; OLIVEIRA, V. D. R. B. \& OLIVEIRA, F. J. A., 1993. Aedes (Stegomyia) aegypti L. e a culicideofauna associada em área urbana da região Sul, Brasil. Revista de Saúde Pública, 27:326-333.

MERCHAN-HAMANN, E., 1997. Diagnóstico macrorregional da situação das endemias das regiões Norte e Nordeste. Informe Epidemiológico do SUS, 6:43-114.

MEDRONHO, R. A., 1995. Geoprocessamento e Saúde: Uma Nova Abordagem do Espaço no Processo Saúde Doença. Rio de Janeiro: Centro de Informação em Ciência e Tecnologia, Fundação Oswaldo Cruz.

MITCHELL, C. J., 1996. Environmental management for vector control. In: The Biology of Disease Vectors (B. J. Beaty \& W. C. Marquardt, eds.), pp. 492501, Niwot: University Press of Colorado.

NOBRE, A.; ANTEZANA, D. \& TAUIL, P. L., 1994. Febre amarela e dengue no Brasil: Epidemiologia e controle. Revista da Sociedade Brasileira de Medicina Tropical, 27 (Sup. 3):59-66.

NOBRE, F. F.; BRAGA, A. L.; PINHEIRO, R. S. \& LOPES, J. A. S., 1996. SIGEPI: Um sistema básico de informação geográfica para apoio à vigilância epidemiológica. Informe Epidemiológico do SUS, 5:5972.

OPS (Organización Panamericana de la Salud), 1995 Dengue y Dengue Hemorrágico en las Américas: Guías para su Prevención y Control. Washington, D.C.: OPS.

REITER, P.; AMADOR, M. A.; ANDERSON, R. A. \& CLARK, G. G., 1995. Short report: Dispersal of Aedes aegypti in urban area after blood feeding as demonstrated by rubidium-marked eggs. American Journal of Tropical Medicine and Hygiene, 52:177-179.
RITCHIE, S. A., 1993. Application of radar rainfall estimates for surveillance of Aedes taeniorhynchus larve. Journal of the American Mosquito Control Association, 9:228-231.

RUSSELL, R. C., 1998. Vectors vs. humans in Australia - Who is on top down under? An update on vector-borne disease and research on vectors in Australia. Journal of Vector Ecology, 23:1-46.

SABROZA, P. C.; TOLEDO, L. M. \& OSANAI, C. H., 1992. A organização do espaço e os processos endêmicos-epidêmicos. In: Saúde, Ambiente e Desenvolvimento. Processos e Conseqüências sobre as Condições de Vida (M. C. Leal, P. C. Sabroza, R. H. Rodriguez \& P. M. Buss, org.), vol. 2, pp. 57-77, São Paulo: Editora Hucitec/Rio de Janeiro: ABRASCO.

SANTOS, S. M., 1999. Homicídios em Porto Alegre, 1996: Análise Ecológica de sua Distribuição e Contexto Socioespacial. Dissertação de Mestrado, Rio de Janeiro: Escola Nacional de Saúde Pública, Fundação Oswaldo Cruz.

SCHULTZ, G. W., 1993. Seasonal abundance of dengue vectors in Manila, Republic of the Philippines. Southeast Asian Journal of Tropical Medicine and Public Health, 24:369-375.

SILVEIRA, A. C., 1998. Dengue: Aspectos epidemiológicos e de controle. Revista da Sociedade Brasileira de Medicina Tropical, 31 (Sup. 2):5-14.

SMITH, T.; CHARLWOOD, J. D.; TAKKEN, W.; TANNER, M. \& SPIEGELHALTER, D. J., 1995. Mapping the densities of malaria vectors within a single village. Acta Tropica, 59:1-18.

SOARES FILHO, B. S. \& SAWYER, D. O., 1996. Correlatos ambientais da distribuição espacial da malária em municípios endêmicos no norte do Estado do Mato Grosso. Informe Epidemiológico do SUS, 5:47-57.

SOUZA-SANTOS, R., 1999. Fatores associados à ocorrência de formas imaturas de Aedes aegypti na Ilha do Governador, Rio de Janeiro, Brasil. Revista da Sociedade Brasileira de Medicina Tropical, 32:373-382.

SPRADLING, S. L.; OLSON, J. K.; COULSON, R. N. \& LOVELADY, C. N., 1998. A geographic information system approach to evaluating the effects of the endangered species protection program on mosquito control. Journal of the American Mosquito Control Association, 14:137-147.

TRPIS, M.; HÄUSERMANN, W. \& CRAIG Jr., G. B., 1995. Estimates of population size, dispersal, and longevity of domestic Aedes aegypti (Diptera: Culicidae) by mark-release-recapture in the Village of Shauri Moyo in Eastern Kenya. Journal of Medical Entomological, 32:27-33.

TUN-LIN, W.; KAY, B. H. \& BARNES, A., 1995. The premisse condition index: A tool for streamlining surveys of Aedes aegypti. American Journal of Tropical Medicine and Hygiene, 53:591-594. 